\title{
Serologic response following SARS-COV2 vaccination in patients with cancer: a systematic review and meta-analysis
}

\author{
Atsushi Sakuraba ${ }^{*}$ (i), Alexander Luna and Dejan Micic
}

\begin{abstract}
Purpose: Patients with cancer have an increased risk of coronavirus disease 2019 (COVID-19) and an attenuated responses to various vaccines. This meta-analysis aims to assess the serologic response to COVID-19 vaccination in patients with cancer.

Methods: Electronic databases were systematically searched on August 1, 2021 for studies that reported the serologic response to COVID-19 vaccine in cancer patients. Random effects models were used to achieve pooled serologic response rates and odds ratios (ORs).

Results: We analyzed 16 observational studies with a total of 1453 patients with cancer. A majority of studies used mRNA vaccines (BNT162b2 or mRNA-1273). The proportion of patients achieving a serologic response after a single and two doses of COVID-19 vaccine were 54.2\% (95\% confidence interval [Cl] 41.0-66.9) and 87.7\% (95\% Cl 82.5-91.5), respectively. Patients with hematologic cancers had a lower response rate after the second dose of vaccine compared to those with solid organ cancers (63.7\% vs. 94.9\%), which was attributable to the low response rates associated with certain conditions (chronic lymphocytic leukemia, lymphoma) and therapies (anti-CD20, kinase inhibitors). A lower proportion of patients with cancer achieved a serologic response compared to control patients after one and two doses of vaccine (OR0.073 [95\% Cl 0.026-0.20] and 0.10 [95\% Cl 0.039-0.26], respectively).
\end{abstract}

Conclusions: Patients with cancer, especially those with hematologic B-cell malignancies, have a lower serologic response to COVID-19 vaccines. The results suggest that cancer patients should continue to follow safety measures including mask-wearing after vaccination and suggest the need for additional strategies for prophylaxis.

Keywords: COVID-19, Vaccine, Outcomes, Meta-analysis, Cancer, Immunocompromised

\section{Introduction}

The coronavirus disease 2019 (COVID-19) pandemic caused by severe acute respiratory syndrome coronavirus 2 (SARS-CoV-2) persists and the world is seeing another year of a global health emergency [1]. Extensive research over the past year has demonstrated that elderly patients as well as those with pre-existing conditions

*Correspondence: asakurab@medicine.bsd.uchicago.edu

Division of Gastroenterology, Hepatology and Nutrition, Department of Internal Medicine, The University of Chicago Medicine, $5841 \mathrm{~S}$

Maryland Ave. MC 4076, Chicago, IL 60637, USA such as obesity, diabetes, and cancer are more susceptible to getting infected with SARS-CoV-2 and developing life threatening pneumonia [2-4]. Approximately $9.5 \%$ of the adult population in the USA have or have had a diagnosis of cancer of any type and 1 in 3 adults will be diagnosed with cancer at some point during their lifetimes [5, 6]. Protecting the health and safety of patients with cancer is vital and considered a major priority during the COVID19 pandemic [7].

Mehta et al. reported that increased age, comorbidities, and poor performance status were associated with poor outcome to COVID-19 in patients with cancer [4]. Case 
fatality rates (CFR) were greater in patients with hematologic cancers compared to those with solid cancers (37\% vs. 25\%) [4]. These data suggest the need for prophylactic strategies in cancer patients, who are often immunosuppressed due to their underlying disease state and use of chemotherapy, radiation therapy, bone marrow or stem cell transplant, and/or immunotherapy. With the lack of effective treatments for COVID-19, prevention strategies including vaccination are of paramount importance in reducing the risk and mortality [8]. Vaccines against COVID-19 were rapidly developed and emerging data shows that mRNA-based COVID-19 vaccines are effective and safe in the general population. However, the efficacy of COVID-19 vaccines in patients with cancer is unknown as patients with active cancer or those treated with immunosuppressing therapies were excluded from regulatory vaccine trials [9]. Guidelines currently recommend that patients with cancer should be vaccinated against SARS-CoV-2 as long as any components of the vaccine are not contraindicated [10]. Data from other vaccine-preventable illnesses suggest an attenuated humoral response to vaccines in patients with solid cancer undergoing cytotoxic chemotherapy [11, 12]. Recent studies have reported that patients with hematologic malignancies, especially those receiving anti-CD20 therapies or those with a history of stem cell transplantation had attenuated immunogenicity to BNT162b2 or mRNA1273 SARS-CoV-2 vaccines compared to those with solid tumors [13, 14]. Additional studies investigating the effectiveness of COVID-19 vaccines in cancer patients are limited and mostly are of small sample sizes.

Better understanding of the overall effectiveness of COVID-19 vaccines in patients with cancer would improve clinical care and protect these vulnerable patient population. In this systematic review and meta-analysis, our aim was to integrate findings across studies to determine the serologic response rate to COVID-19 vaccination in patients with cancer.

\section{Methods}

\section{Search strategy and study selection}

This meta-analysis was conducted according to a priori defined protocol that is in accordance with the PRISMA guideline [15]. The protocol of this meta-analysis has been submitted to the International Prospective Register of Systematic Reviews (PROSPERO) [16]. We searched PubMed/MEDLINE, EMBASE, and medRxiv (https:// www.medrxiv.org/) from inception to August 1, 2021 to identify studies assessing the response to COVID-19 vaccination in patients with cancer.

We included observational studies reporting the outcomes of COVID-19 vaccination in patients with cancer. There were no restrictions regarding age, sex or duration of the study. Studies reporting outcomes in patients with active or history of cancer were eligible. We imposed no geographic or language restrictions. Two authors (AL, AS) independently screened each of the potential studies to determine whether they were eligible for inclusion. Areas of disagreement or uncertainty were resolved by consensus among the authors. Studies were identified with the following terms:"COVID-19", "SARS-CoV-2", "vaccine", "cancer", "malignancy", "leukemia", "lymphoma", "immunosuppressed", and "hematologic diseases". A search was also performed of bibliographies of identified articles for additional references. The search was restricted to human studies. Manuscripts published in languages other than English were translated if necessary. Single case reports were excluded. Studies that reported only adverse outcomes to COVID-19 vaccination were excluded. The corresponding authors of studies were contacted to obtain additional information when needed. The search strategy is described in Fig. 1 and the Pubmed/MEDLINE, EMBASE, and medRxiv search strategies are shown in Additional file 1: Table S1.

\section{Data extraction and quality assessment}

All data were independently abstracted in duplicate by two authors (AL, AS) by using a data extraction form. Data on the study characteristics including author name, year of publication, study design, duration, study location, sample size, diagnosis of cancer, concomitant medication use, age and gender of patients, type and frequency of vaccination, and type and outcome of serologic testing were collected. We divided diseases into the following two categories: (1) solid tumors, and (2) hematologic malignancies. Proportion of patients undergoing active cancer therapies and using glucocorticoids (GCs) were extracted when data was available.

We assessed the risk of bias of included studies using the Joanna Briggs Institute Critical Appraisal Checklist [17]. We rated the quality of evidence according to the Grades of Recommendation, Assessment, Development and Evaluation (GRADE) approach to assess the certainty of evidence obtained from the present meta-analysis [18].

\section{Outcome assessment}

The primary outcome was the rate of serologic response to COVID-19 vaccination in cancer patients. Response was separately assessed after one or two vaccinations. The secondary outcome of interest was the rate of serologic response in cancer patients compared to control patients. We extracted the number of patients who achieved an above cut-off antibody level among the total number of patients tested in each study. Applying a common cutoff value between studies was not possible because each study used a different serologic testing method. When 


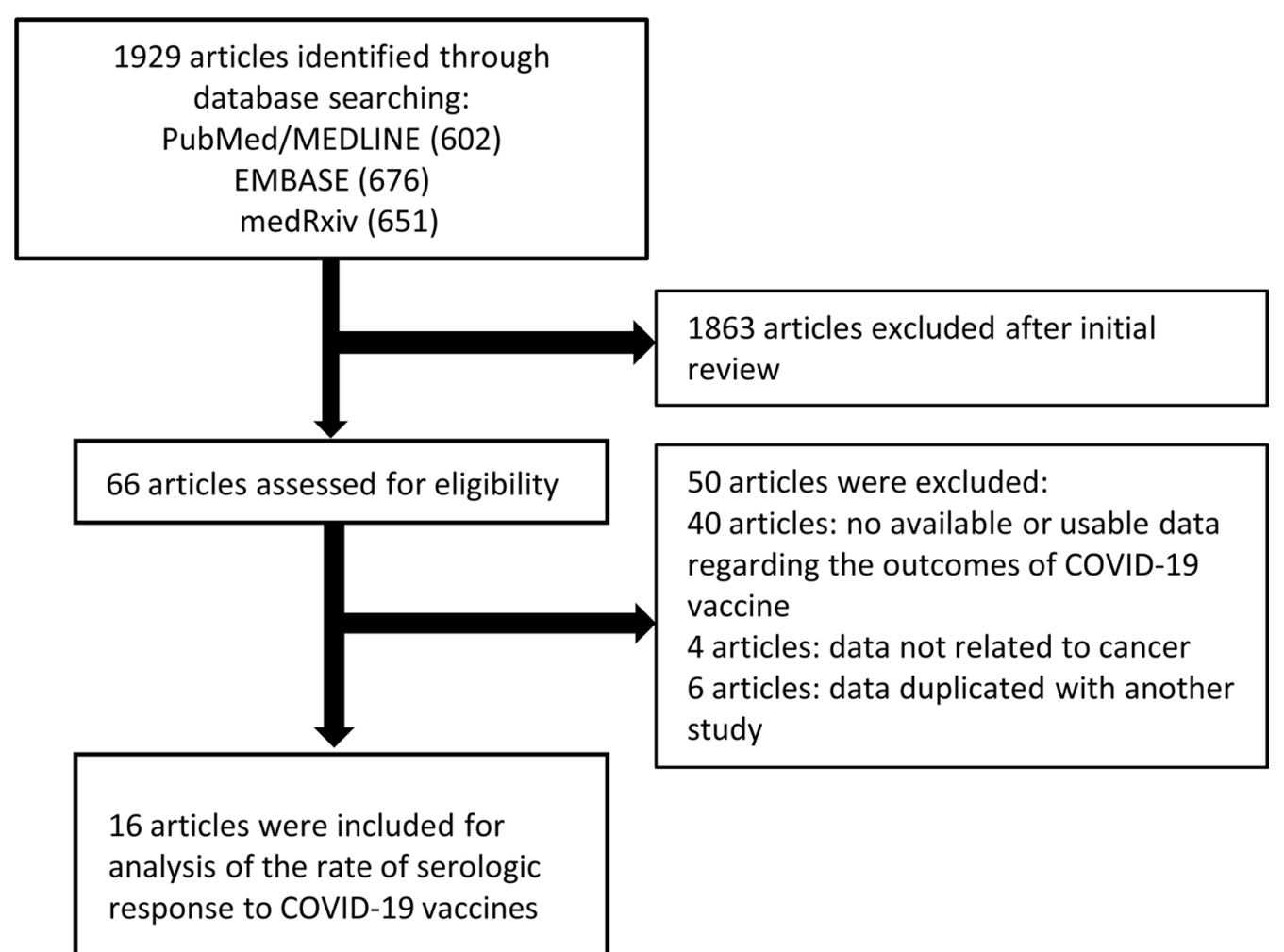

Fig. 1 Flowchart of the assessment of the studies identified in the meta-analysis

tests were performed at multiple time points in a study, we chose the date closest to 4 weeks after the vaccination.

Subgroup analyses or meta-regression according to cancer type (hematological vs solid cancer), proportion of patients on anti-cancer medication/treatment, and age of subjects were undertaken when data were available. Anti-cancer medications/treatments included chemotherapy, monoclonal antibodies, immune check-point inhibitors, radiotherapy, hormonal therapy, etc. and were assessed separately.

\section{Statistical analysis}

We undertook a meta-analysis of the rate of serologic response to COVID-19 vaccination among individuals with cancers from observational studies by using a random effects model. The presence of heterogeneity across studies was assessed by using the $I^{2}$ statistic. An $I^{2}$ value of $<25 \%$ indicates low heterogeneity, $25-75 \%$ as moderate heterogeneity and $>75 \%$ as considerable heterogeneity [19]. Heterogeneity was evaluated by using Cochran's Q-statistics with a significance level of $P<0.10$ [20]. Begg's and Egger's tests were performed to assess publication bias and funnel plots were constructed to visualize asymmetry when $\geq 3$ studies were available [21, 22]. A random effects meta-regression model was used to assess the contributions of each potential risk factors and medication class to the outcome of vaccine response. When the number of available studies for each analysis was less than 10, funnel plot construction and meta-regression analysis were undertaken for reference purpose due to its low reliability.

We included preprints because they form a substantial part of the available COVID-19 evidence, but due to their lack of peer-review, we conducted a sensitivity analysis by excluding preprints [23]. Four studies included non-mRNA type vaccines, so we conducted a sensitivity analysis by excluding them. We also performed one study removed analyses to assess whether the results are strongly influenced by any single study.

Statistical analyses were performed using the Comprehensive Meta Analysis Software (version 3; Biostat, Englewood, NJ, USA). All statistical tests except for the Q-statistics used a two-sided $P$ value of 0.05 for significance.

\section{Data sharing and access}

Data will be made available upon request to the corresponding author. All authors had access to the study data and reviewed and approved the final manuscript. 


\section{Results}

\section{Study characteristics}

We identified 1929 citations through the literature search, excluded 1863 after initial title and abstract screening, and assessed 66 studies for eligibility. Sixteen articles including 1453 patients met eligibility criteria (Fig. 1). As shown in Table 1, seven were full-text articles $[13,14$, 24-28], seven were correspondence/letters [24, 29-34], and two were articles in a preprint $[35,36]$. Four studies included only patients with solid organ cancers, nine included only hematologic cancers, and three studies included patients with both solid and hematologic cancers. The three studies that included both types of cancers reported seroconversion rates separately among the types of cancers, so we reported them separately. Eight studies used only BNT162b2 (Pfizer-BioNTech), four studies used BNT162b2 and mRNA-1273 (Moderna), two studies used ChAdOx1 nCoV-19 (Oxford-AstraZeneca) and BNT162b2, one study used ChAdOx1 nCoV-19, mRNA-1273, and BNT162b2, and one study used AD26. COV2.S (Janssen/Johnson \& Johnson), mRNA-1273, and BNT162b2. The majority of the studies that used nonmRNA type vaccines did not report outcomes separately, so we reported them together with the mRNA vaccination strategies.

For the analyses on the rate of serologic response to COVID-19 vaccination, nine and eleven studies were available for assessment after one and two doses, respectively. Six and eight studies compared outcomes after one or two doses of COVID-19 vaccine to a control population without cancers. A majority of the studies assessed serologic response 3-5 weeks post-vaccination. All studies included for the assessment of response after two doses did not delay the timing of the second dose. The summary of characteristics and outcomes of the included studies are summarized in Table 1 . The risk of bias of included studies assessed using the Joanna Briggs Institute Critical Appraisal Checklist is shown in Additional file 1: Table S2. A majority of the studies were of medium to high quality.

\section{Rate of serologic response after a single dose of COVID-19 vaccine}

There were nine studies (11 reports) that assessed the serologic response after the first dose of COVID19 vaccine in patients with cancer $[14,24,25,27,29$, 32-34] The studies by Monin et al. and Addedo et al. reported outcomes separately in hematologic and solid cancers $[14,25]$. As shown in Fig. 2A, the pooled proportion of patients achieving a serologic response was $54.2 \%$ (95\% confidence interval [CI] 41.0-66.9).
Subgroup analysis demonstrated that the rate was numerically lower in solid cancers $(49.6 \%)$ compared to hematologic cancers (56.0\%).

Heterogeneity was present $\left(I^{2}=88.9 \%\right)$ likely attributed to the difference in the reported rates among studies. Multivariate meta-regression was undertaken to further explore the cause of heterogeneity and demonstrated that the proportion of patients on anti-cancer therapy (coefficient-0.029, 95\% $\mathrm{CI}-0.055-(-0.0016), P=0.038)$ and the age of the subjects (coefficient $-0.10,95 \% \mathrm{CI}-0.16-(-0.050)$, $P<0.001)$ were significant sources of heterogeneity, with studies with more patients on anti-cancer therapy and with older subjects presenting a lower serologic response rates to a single dose of COVID-19 vaccine (Additional file 1: Table S3).

Funnel plot of the studies included in the meta-analysis demonstrated no asymmetry and no publication bias was found (Begg's $P=0.44$, Egger's $P=0.48$ ) (Additional file 1: Fig. S1A).

Sensitivity analyses were carried out to assess whether individual studies influenced the results (Additional file 1: Fig. S1B). When individual studies were removed one at a time from the analyses, the corresponding pooled rates were not markedly altered by any single study confirming the stability of the results. Three studies included non-mRNA type vaccines $[29,32,34]$ and a sensitivity analysis excluding them showed similar results (Additional file 1: Fig. S1C). No preprint studies were included for this analysis, so sensitivity analysis excluding preprints was not performed.

Subgroup analysis according to type of hematologic cancer demonstrated that rates were lower in chronic lymphocytic leukemia (CLL) (16.7\%), and lymphoma (16.3\%) compared to multiple myeloma (MM) (36.8\%), myeloproliferative malignancies (MPM) (54.6\%), and chronic myeloid leukemia (CML) (72.2\%) (Additional file 1: Fig. S1D). In solid cancers, the rates were lower in thoracic cancers $(21.4 \%)$ and skin cancers $(22.2 \%)$ compared to women's cancers $(47.4 \%)$, gastrointestinal (GI) cancers $(50.0 \%)$, and urological cancers $(66.7 \%)$ (Additional file 1: Fig. S1E). Subgroup analysis according to type of vaccine showed that the rate was higher with mRNA-1273 (80.4\%) compared to BNT162b2 (53.0\%) and AZD1222 (58.5\%) (Additional file 1: Fig. $\mathrm{S} 1 \mathrm{~F})$. Subgroup analysis according to type of therapy demonstrated that the rate was lowest with anti-CD20 therapy (10.0\%) compared to chemotherapy (55.0\%), kinase inhibitors (61.6\%), immune check-point inhibitors $(84.6 \%)$, or no therapy (71.5\%) (Additional file 1: Fig. S1G). 


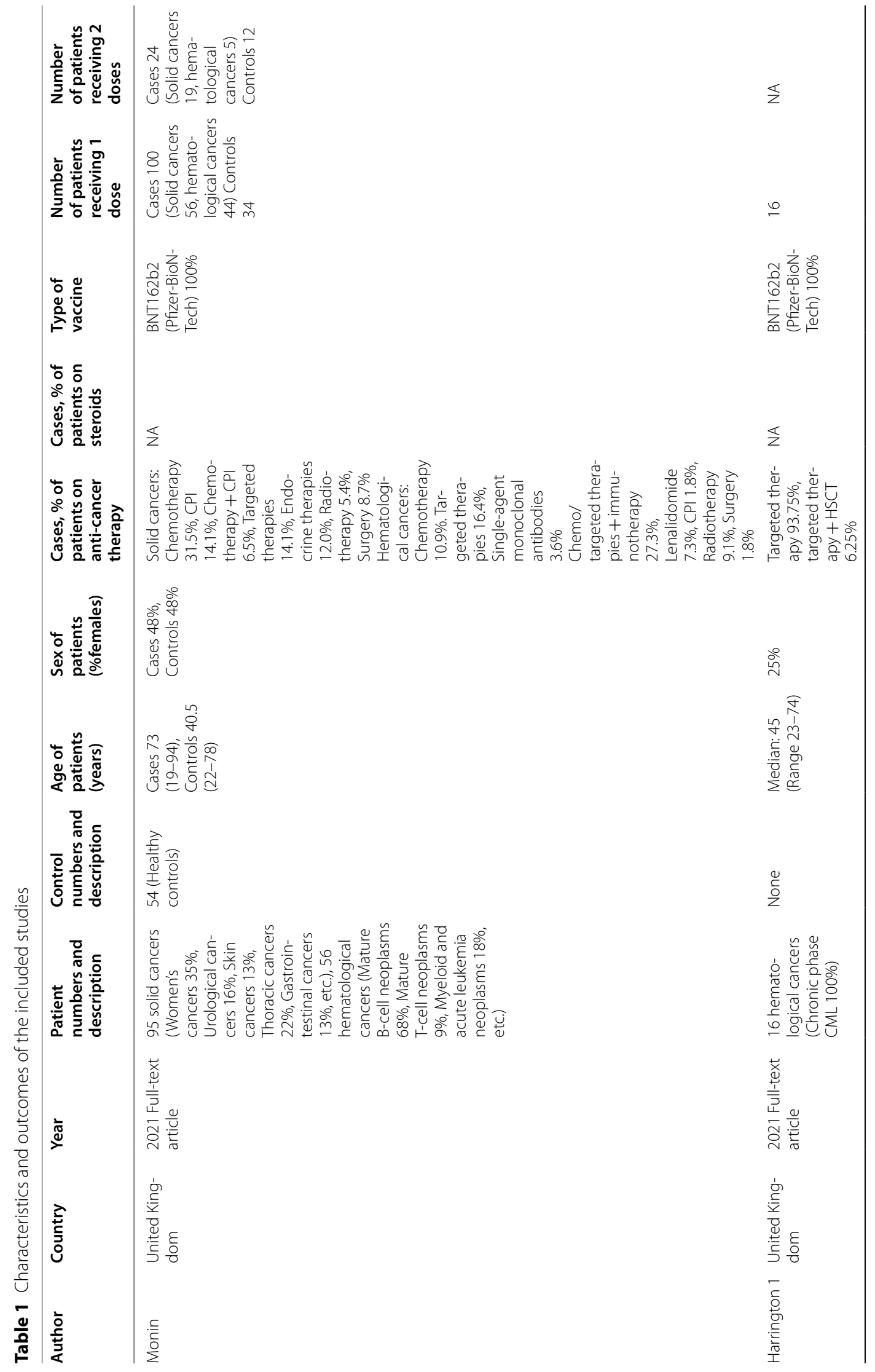




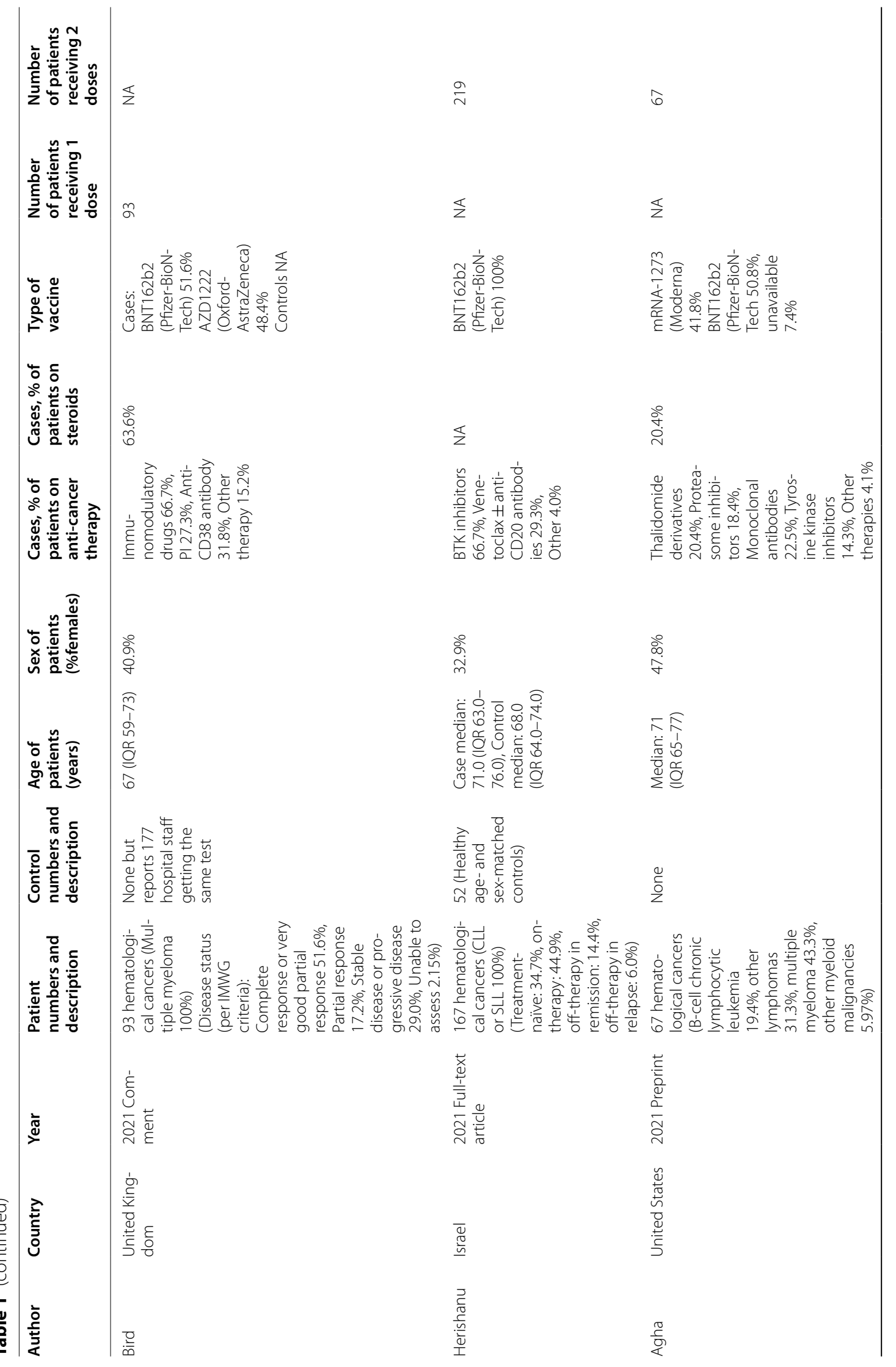




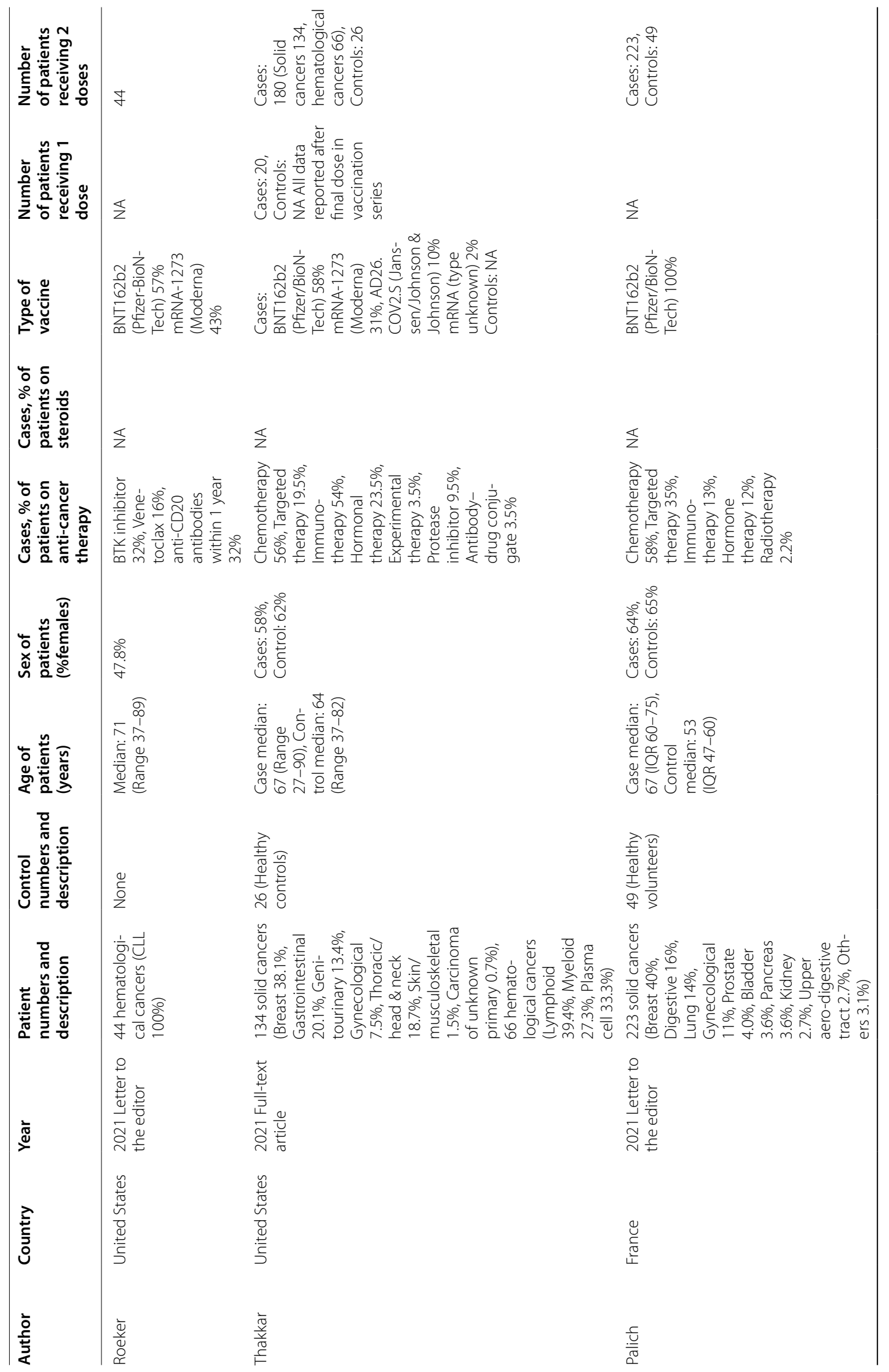




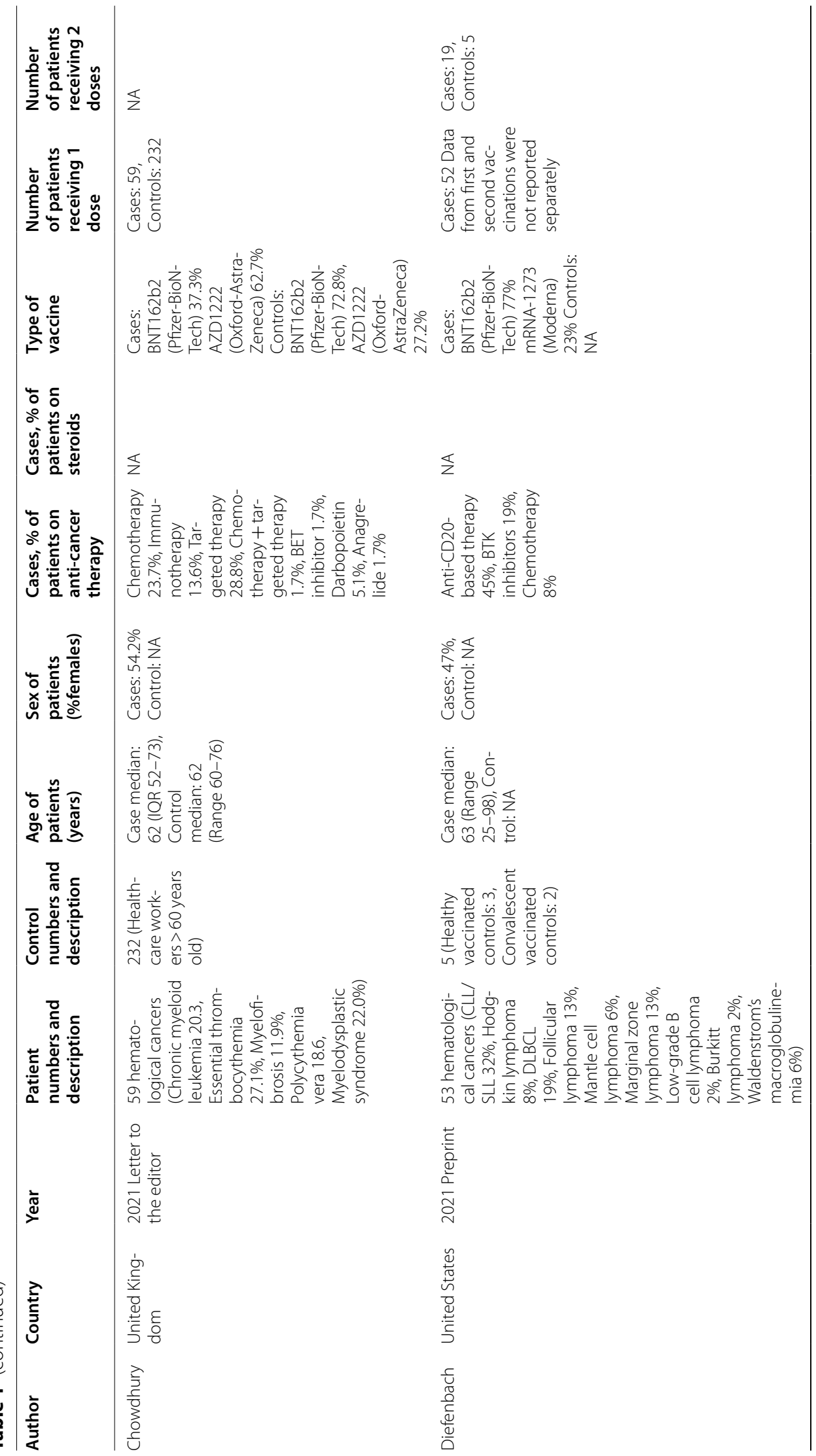




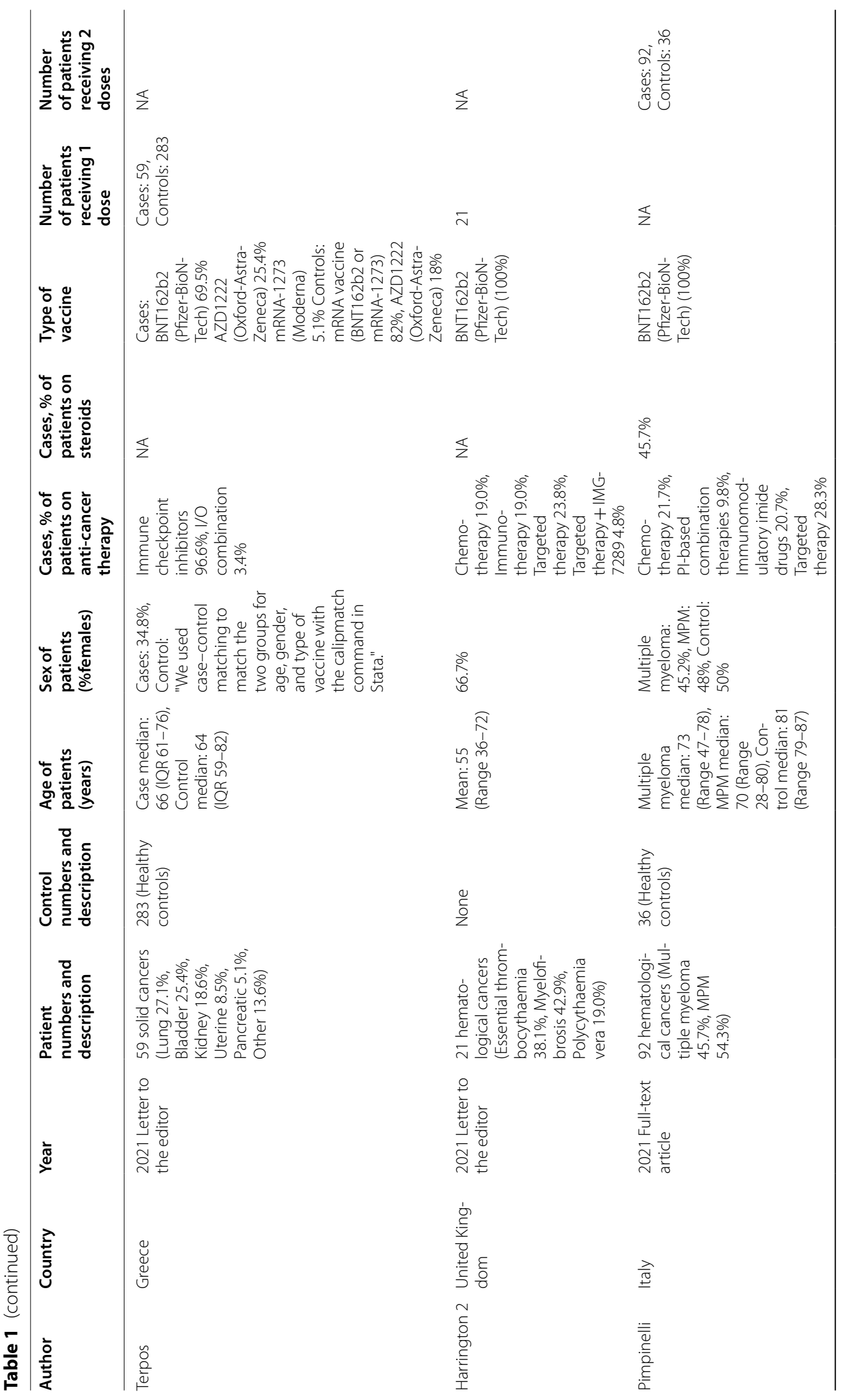




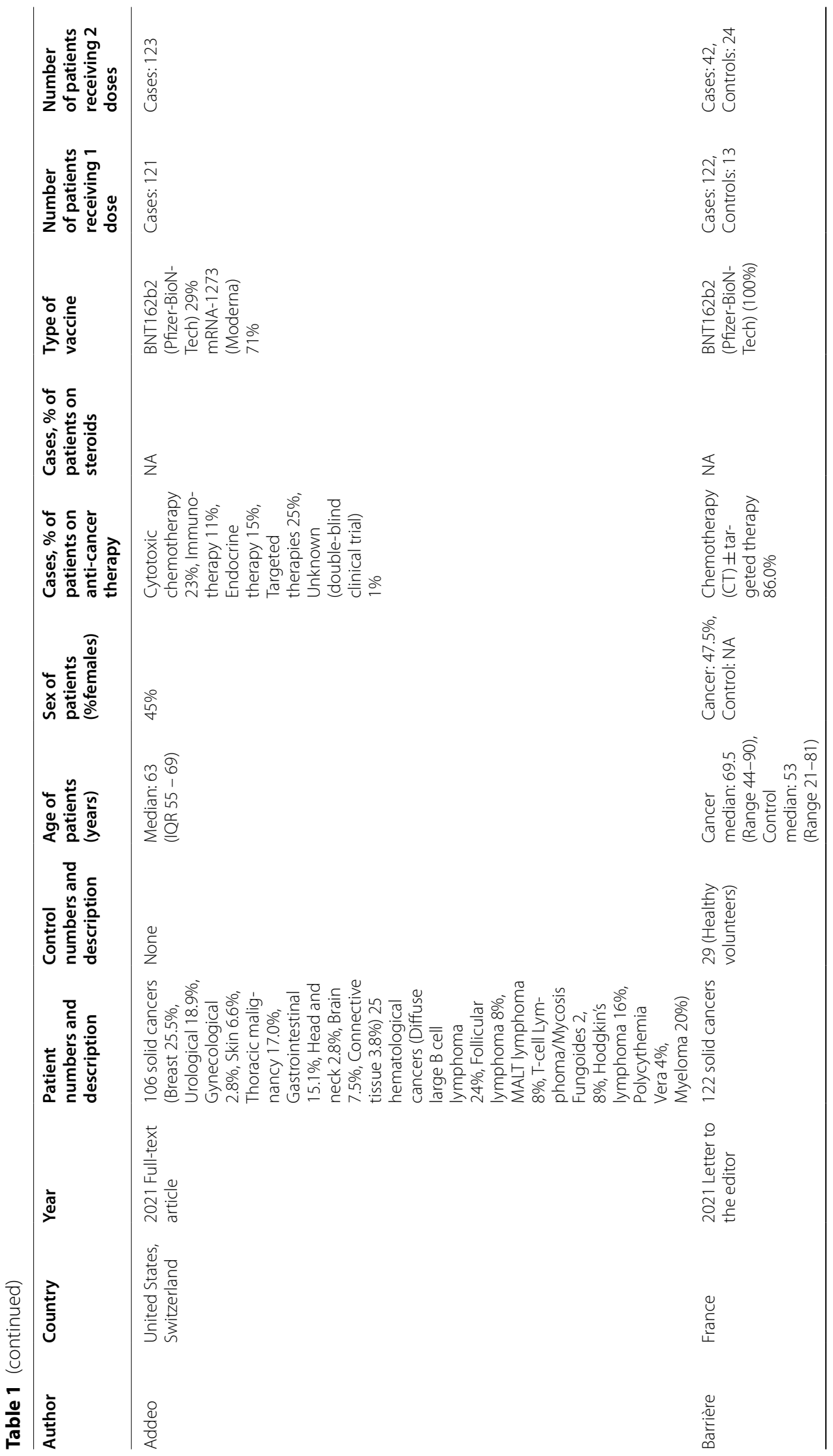




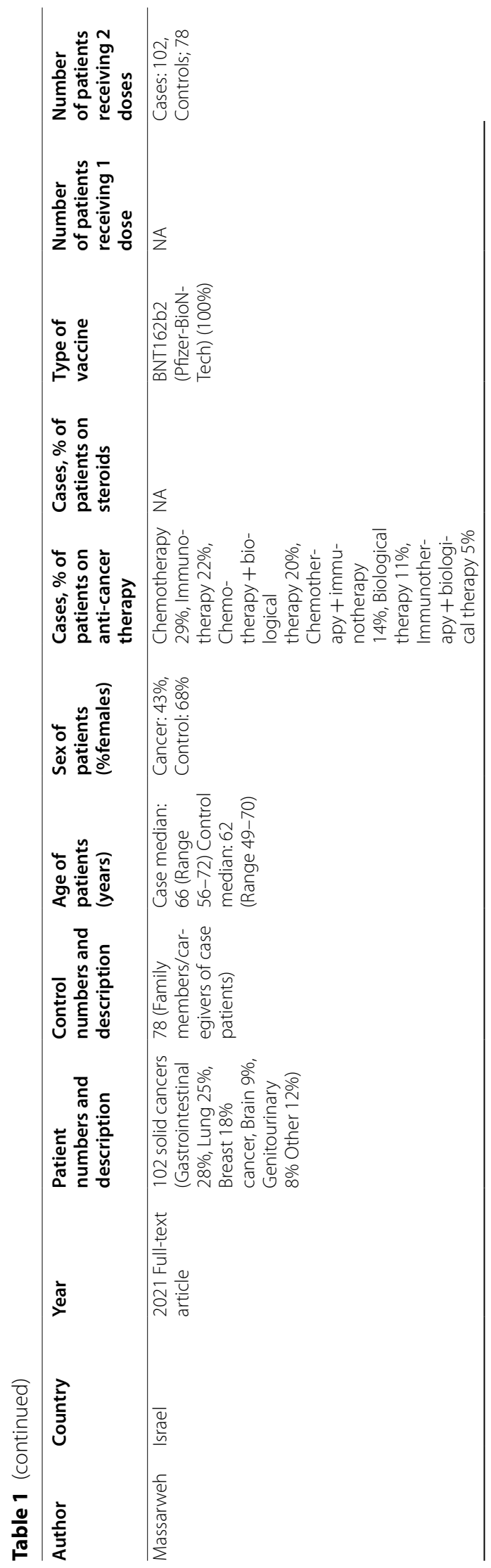




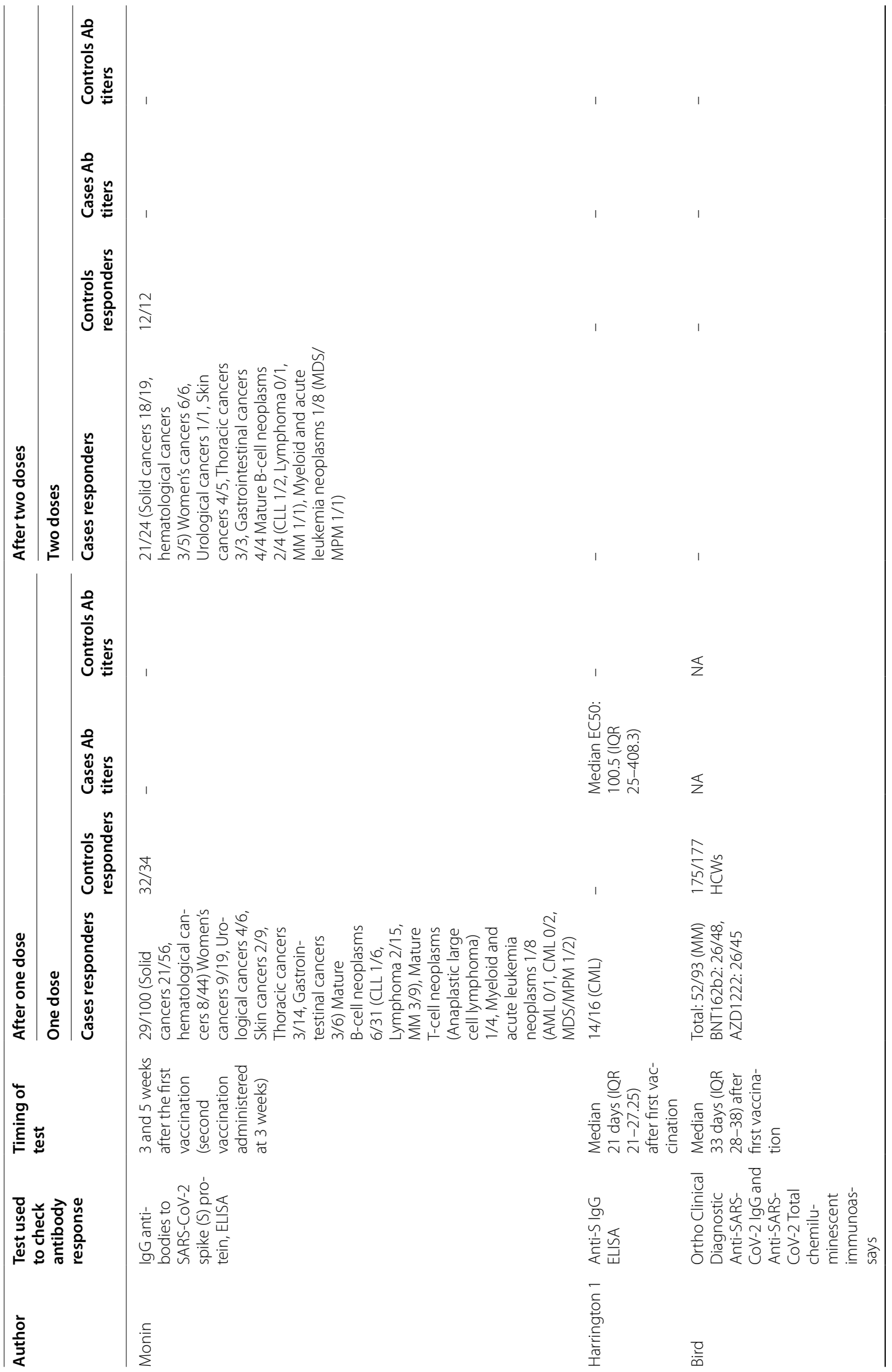




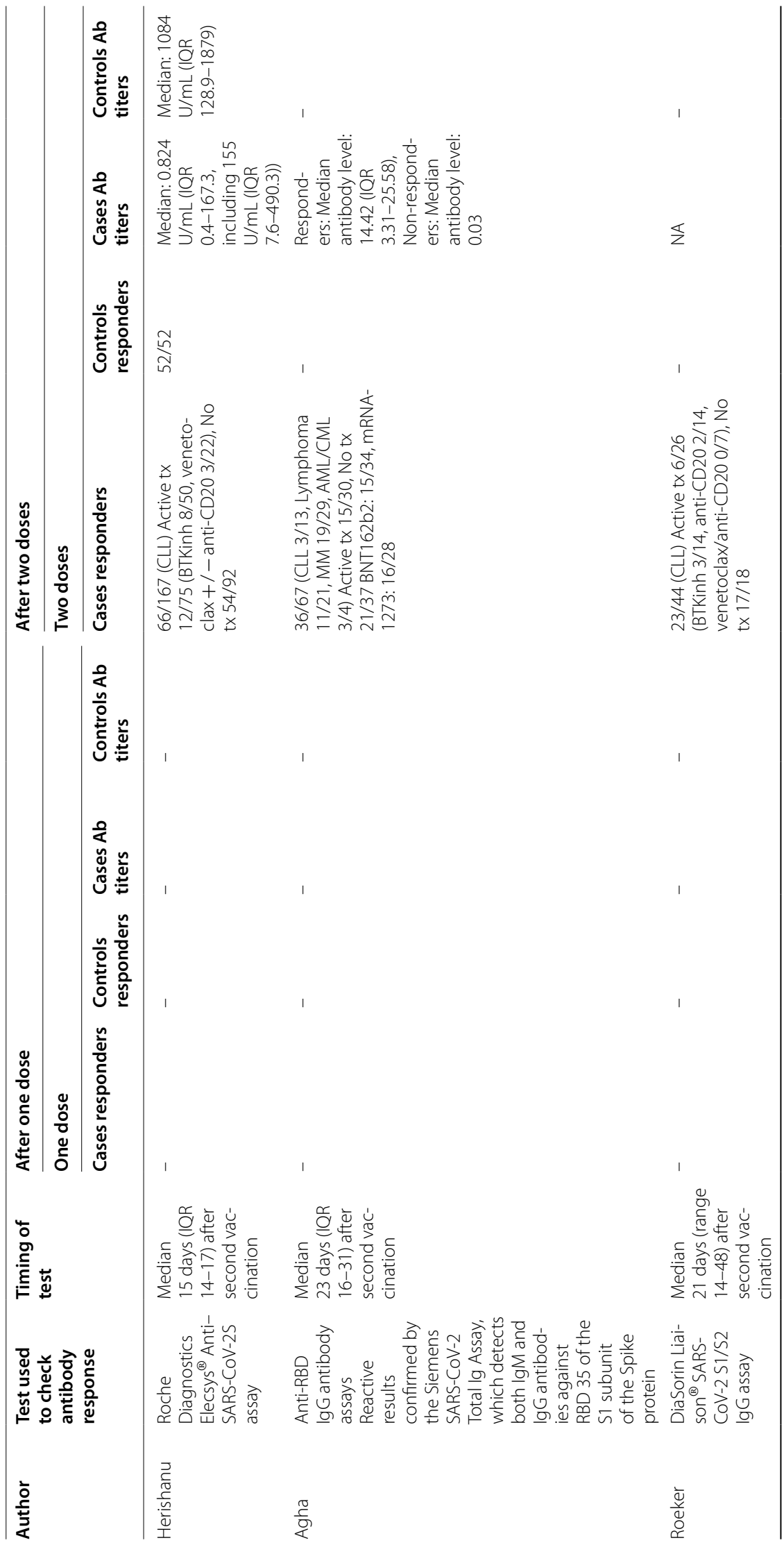




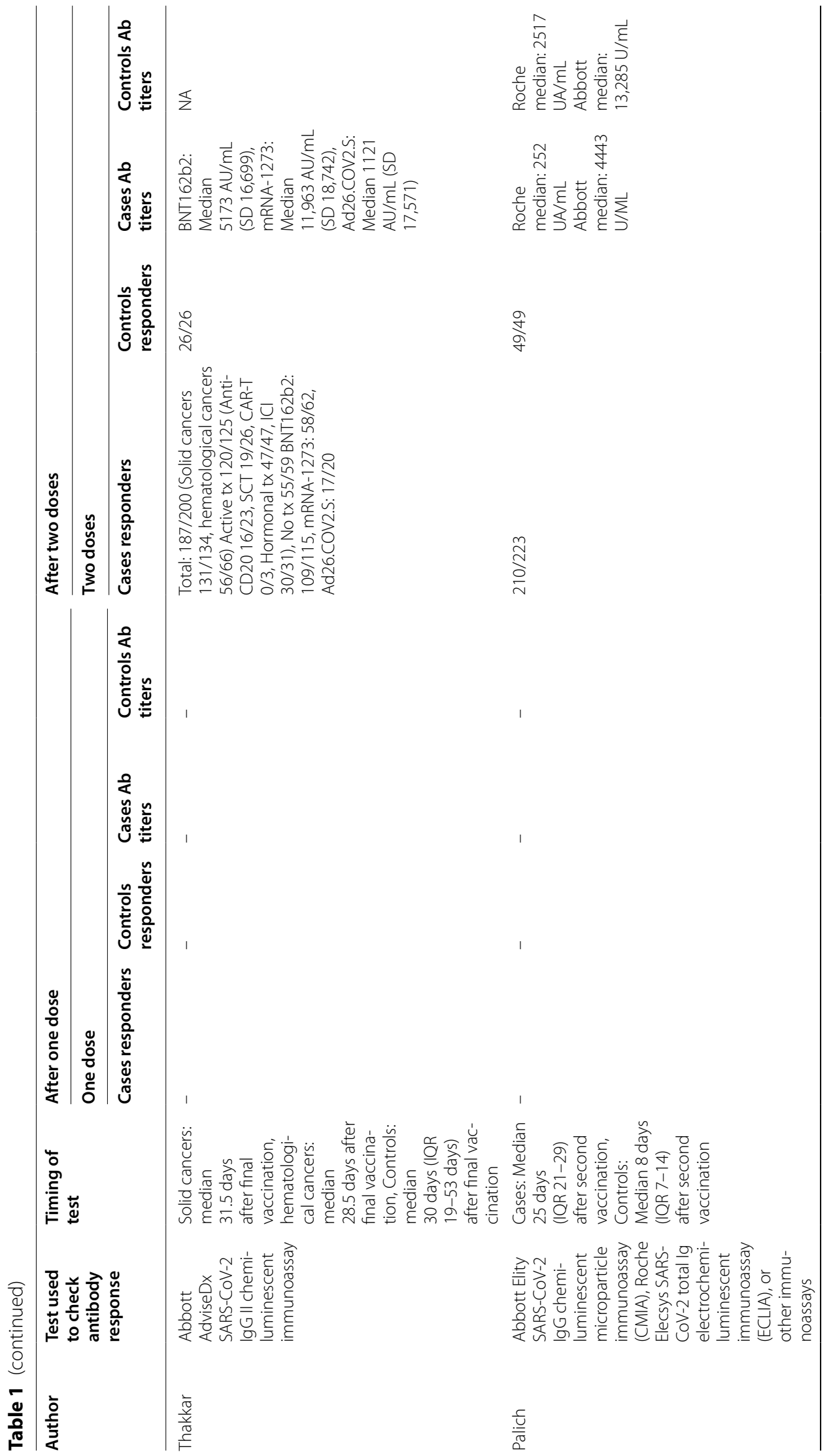




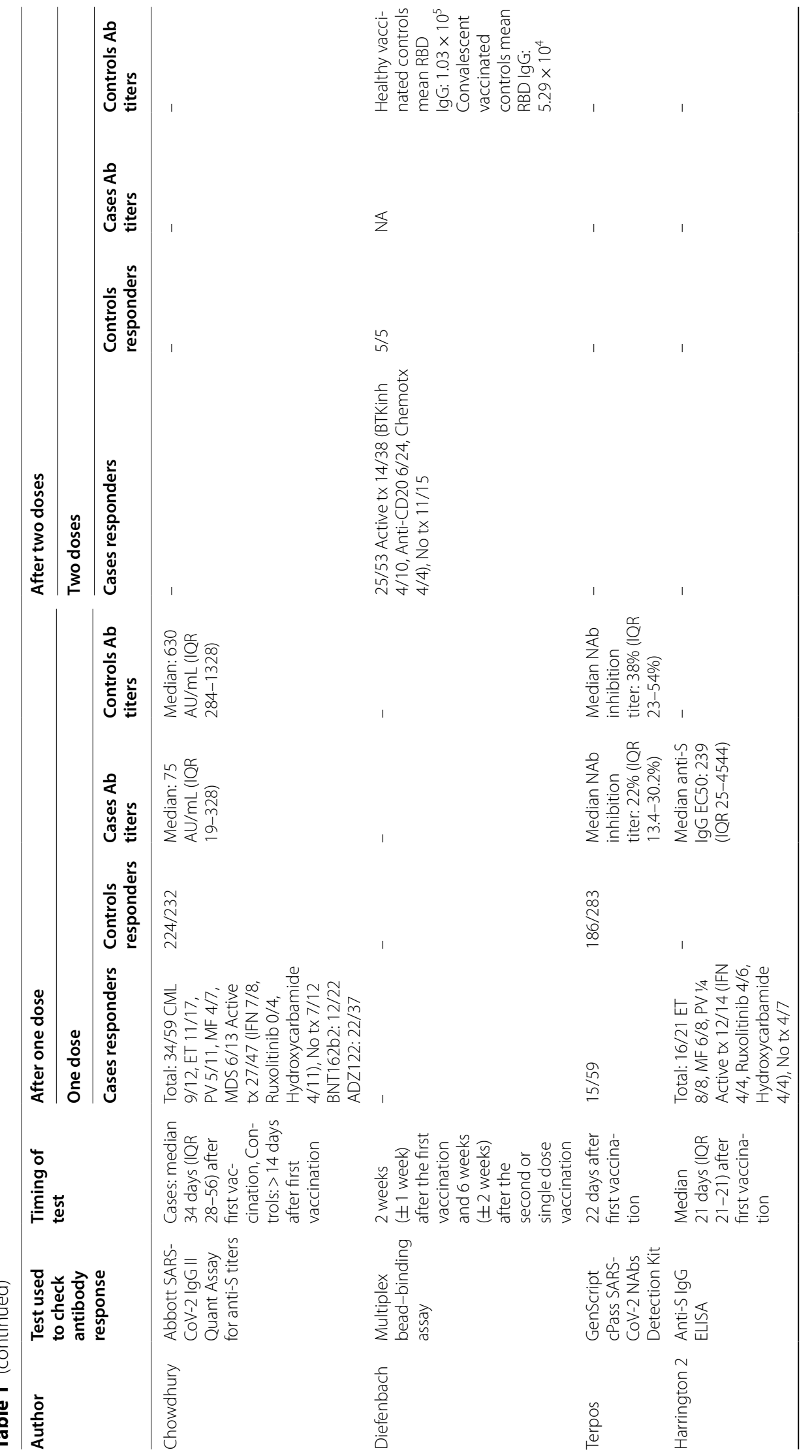




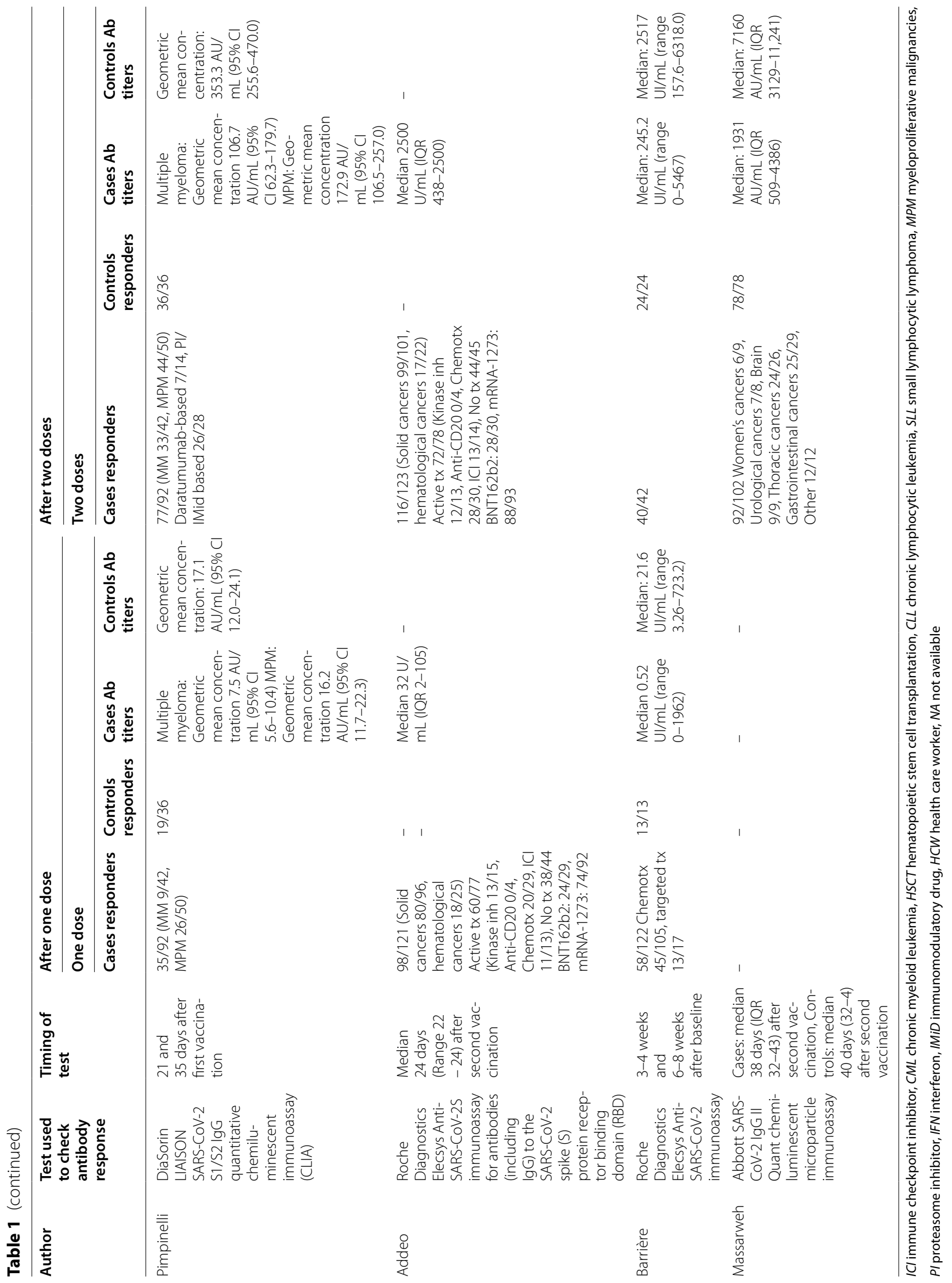




\section{Rate of serologic response after two doses of COVID-19 vaccine}

There were eleven studies (14 reports) that assessed the serologic response after two doses of COVID-19 vaccine $[13,14,25-28,30,31,33,35,36]$. All studies did not delay the timing of the second dose including the one study reported from United Kingdom [25]. Three studies reported outcomes separately in hematologic and solid cancers $[13,14,25]$. As shown in Fig. 2B, the pooled proportion of patients achieving a serologic response was $87.7 \%$ (95\% CI 82.5-91.5). Subgroup analysis demonstrated that the rate was numerically lower in hematologic cancers (63.7\%) compared to solid cancers (94.9\%). Surprisingly, the rates in patients with hematologic cancers after one and two doses were not considerably different $(59.0-63.7 \%)$.

Heterogeneity was present $\left(I^{2}=94.2 \%\right)$ likely attributed to the difference in the reported rates between hematologic and solid cancers. Multivariate meta-regression was undertaken to further explore the cause of heterogeneity and demonstrated that the difference in study population (hematologic versus solid cancer) (coefficient - 2.16, 95\% $\mathrm{CI}-3.26-(-3.86), P<0.001)$ was a significant source of heterogeneity (Additional file 1: Table S4).

Funnel plot of the studies included in the meta-analysis demonstrated no asymmetry, but publication bias was present by Egger's $(P=0.014)$ but not Begg's test $(P=0.27)$ (Additional file 1: Fig. S2A).

Sensitivity analysis was undertaken by excluding two preprint studies (Additional file 1: Fig. S2B). Exclusion of preprints demonstrated a serologic response rate similar to when they were included ( $87.7 \%$ vs. $91.6 \%)$. Remove one study analysis also showed that pooled rates were not markedly altered by any single study (Additional file 1: Fig. S2C). Excluding one study that included nonmRNA vaccines [13] demonstrated similar results (data not shown).

Similar to the results after one dose, subgroup analysis stratified by type of hematologic cancer demonstrated that rates were lower among conditions that mainly affect B-cells, such as CLL (41.9\%), and lymphoma (52.4\%), compared to MM (72.7\%), AML/CML (75.0\%), and MPM (88.0\%) (Additional file 1: Fig. S2D). In solid cancers, the rates were slightly lower in women's cancers (76.6\%) and skin cancers $(80.0 \%)$ compared to GI cancers $(86.7 \%)$, urological cancers (87.5\%), thoracic cancers (91.5\%), and brain cancers (95.0\%) (Additional file 1: Fig. S2E). Subgroup analysis according to type of vaccine showed that the rates were similar with mRNA-1273 (87.2\%) and BNT162b2 (85.1\%) (Additional file 1: Fig. S2F). Subgroup analysis according to type of therapy demonstrated that the rates were lower with chimeric antigen receptor (CAR) T-cell therapy (12.5\%), anti-CD20 therapy (22.9\%), kinase inhibitors (38.9\%), daratumab (50.0\%), and stem cell transplant (SCT) (73.1\%) compared to chemotherapy (92.8\%), protease inhibitors (92.9\%), immune check-point inhibitors (95.2\%), hormonal therapy $(99.0 \%)$, or no therapy (82.3\%) (Additional file 1: Fig. S2G).

\section{Comparison of serologic response after a single dose of COVID-19 vaccine to controls}

As shown in Fig. 3A, meta-analysis of 6 studies (7 reports) $[25,27,29,32-34]$ that included control patients demonstrated that a significantly lower proportion of cancer patients achieved a serologic response compared to control patients after a single dose of vaccine (odds ratio (OR) $0.073,95 \%$ CI $0.026-0.20, P<0.001$ ). Subgroup analysis showed that both hematologic and solid cancers demonstrated lower response rates compared to controls (OR 0.052, 95\% CI 0.008-0.33, $P=0.0016$ and OR 0.085, 95\% CI 0.024-0.29, $P<0.001$, respectively).

Heterogeneity was present $\left(I^{2}=84.3 \%\right)$ likely attributed to the variable ratios reported among included studies (range 0.014-0.55), especially among studies included in hematologic cancers. Funnel plot of the studies included in the meta-analysis demonstrated no asymmetry (Begg's $P=0.55$, Egger's $P=0.099$ ) (Additional file 1: Fig. S3A).

\section{Comparison of serologic response after two doses of COVID-19 vaccine to controls}

As shown in Fig. 3B, meta-analysis of 8 studies (10 reports) [13, 25-28, 31, 33, 36] that included control patients demonstrated that a significantly lower proportion of cancer patients achieved a serologic response compared to control patients after two doses of vaccine (OR 0.10 (95\% CI 0.039-0.26), $P<0.001$ ). Subgroup analysis showed that both hematologic and solid cancers demonstrated lower response rates compared to controls (OR 0.044, 95\% CI 0.012-0.16, $P<0.001$ and OR 0.24, 95\% CI $0.062-0.90, P=0.035$, respectively), but the OR was much smaller in hematologic cancer.

Heterogeneity was not present $\left(I^{2}=0 \%\right)$ and funnel plot of the studies included in the meta-analysis demonstrated no asymmetry (Begg's $P=0.15$, Egger's $P=0.15$ ) (Additional file 1: Fig. S3B).

Some studies reported data of absolute values of antibody titers (Table 1). Cancer patients had values that were $1 / 3$ to $1 / 10$ compared to controls.

\section{Grading the quality of evidence}

Based on the GRADE approach, an overall quality of evidence for this analysis was low as the data were obtained from observational studies and there were no specific factors to down- or up-grade the level of certainty (Additional file 1: Table S5). 


\section{A. Serologic response after one dose of vaccine}

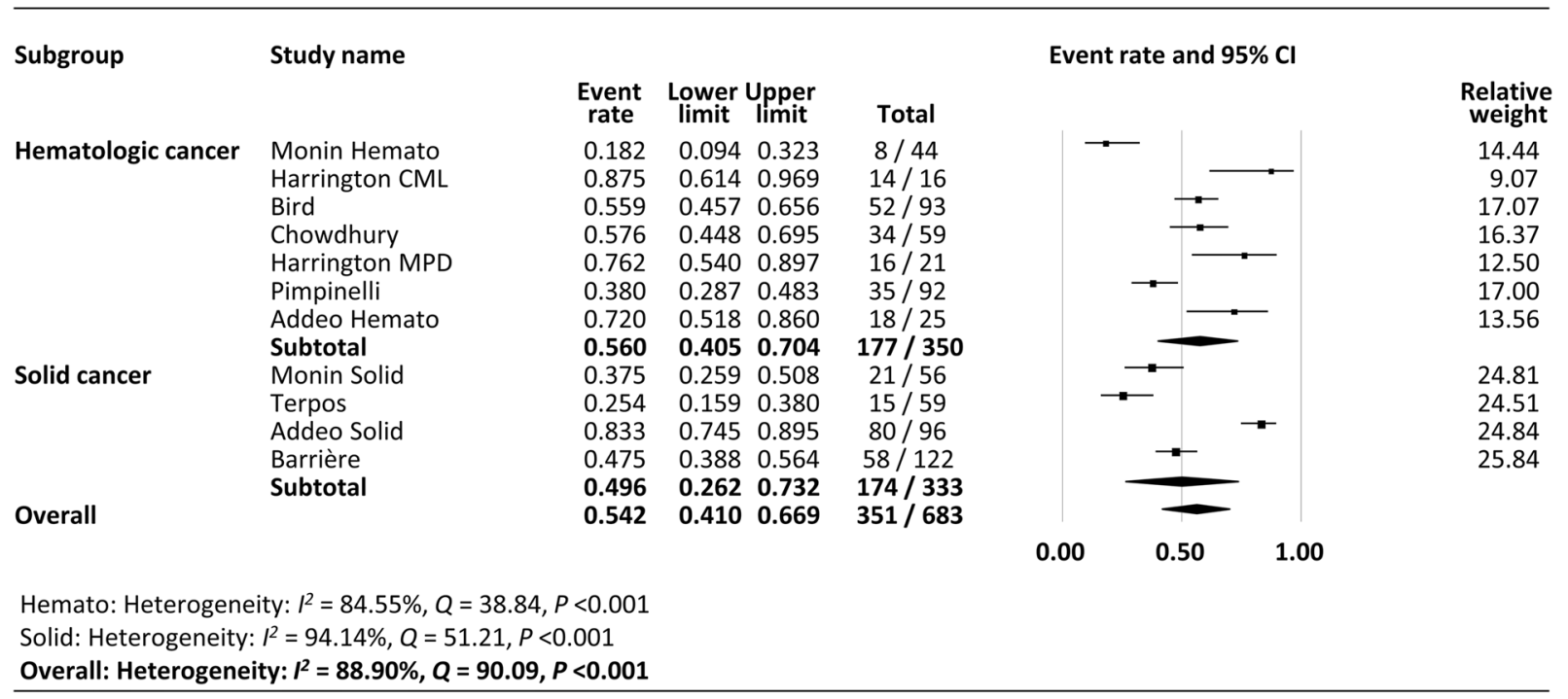

\section{B. Serologic response after two doses of vaccine}

$\begin{array}{llllll}\text { Subgroup } & \text { Study name } & & & \\ \text { Hematologic cancer } & \text { Monin Hemato } & \begin{array}{c}\text { Event } \\ \text { rate }\end{array} & \begin{array}{c}\text { Lower Upper } \\ \text { limit }\end{array} & \text { limit } & \text { Total } \\ & \text { Herishanu } & 0.600 & 0.200 & 0.900 & 3 / 5 \\ & \text { Agha } & 0.395 & 0.324 & 0.471 & 66 / 167 \\ & \text { Roeker } & 0.537 & 0.418 & 0.652 & 36 / 67 \\ & \text { Thakkar Hemato } & 0.523 & 0.377 & 0.664 & 23 / 44 \\ & \text { Diefenbach } & 0.848 & 0.741 & 0.916 & 56 / 66 \\ & \text { Pimpinelli } & 0.472 & 0.342 & 0.605 & 25 / 53 \\ \text { Solid cancer } & \text { Addeo Hemato } & 0.837 & 0.747 & 0.902 & 77 / 92 \\ & \text { Subtotal } & 0.773 & 0.556 & 0.902 & 17 / 22 \\ & \text { Monin Solid } & \mathbf{0 . 6 3 7} & \mathbf{0 . 4 8 1} & \mathbf{0 . 7 6 9} & \mathbf{3 0 3 / 5 1 6} \\ & \text { Thakkar Solid } & 0.947 & 0.706 & 0.993 & 18 / 19 \\ & \text { Palich } & 0.978 & 0.933 & 0.993 & 131 / 134 \\ & \text { Addeo Solid } & 0.942 & 0.902 & 0.966 & 210 / 223 \\ \text { Overall } & \text { Barrière } & 0.980 & 0.924 & 0.995 & 99 / 101 \\ & \text { Massarweh } & 0.952 & 0.829 & 0.988 & 40 / 42 \\ & \text { Subtotal } & 0.902 & 0.827 & 0.946 & 92 / 102 \\ & & \mathbf{0 . 9 4 9} & \mathbf{0 . 9 1 6} & \mathbf{0 . 9 7 0} & \mathbf{5 9 0} / \mathbf{6 2 1} \\ & & \mathbf{0 . 8 7 7} & \mathbf{0 . 8 2 5} & \mathbf{0 . 9 1 5} & \mathbf{8 9 3} / \mathbf{1 1 3 7}\end{array}$

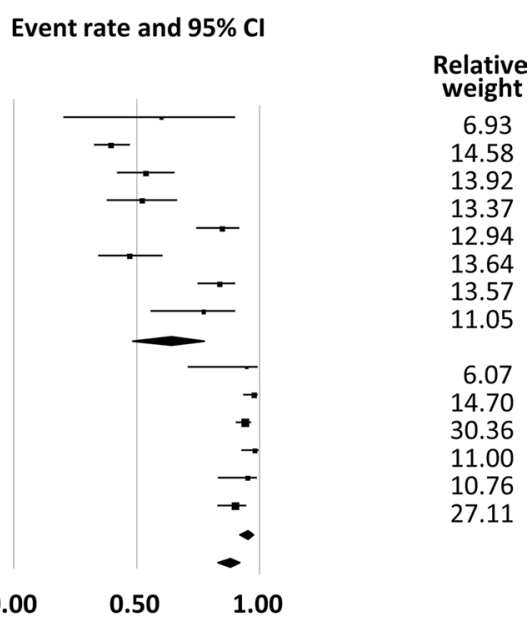

Hemato: Heterogeneity: $I^{2}=89.49 \%, Q=66.59, P<0.001$

Solid: Heterogeneity: $P^{2}=39.79 \%, Q=8.31, P=0.14$

Overall: Heterogeneity: $I^{2}=94.22 \%, Q=224.81, P<0.001$

Fig. 2 A Meta-analysis of serological response after one dose of vaccine. B Meta-analysis of Serological response after two doses of vaccine

\section{Discussion}

In the present meta-analysis, we assessed the serologic response to COVID-19 vaccination in patients with cancer. We demonstrated that only $54 \%$ of patients with cancer achieved a serologic response to a single dose of COVID-19 vaccine, which improved to $88 \%$ after two doses. The rates were significantly lower compared to controls at both stages, especially in patients with hematologic cancers, suggesting the urgent need for an improved vaccination strategy in this vulnerable patient population. Further studies assessing the response in patients with various types of cancers or to other types of vaccine are warranted.

Patients with cancer are known to have a greater mortality due to COVID-19 [37]. Patients with cancer are immunocompromised due to the immunosuppressive properties of cancer and the effects of chemotherapy and radiation therapy [38]. Chemotherapy may cause 


\section{A. Serologic response compared to controls after one dose of vaccine}

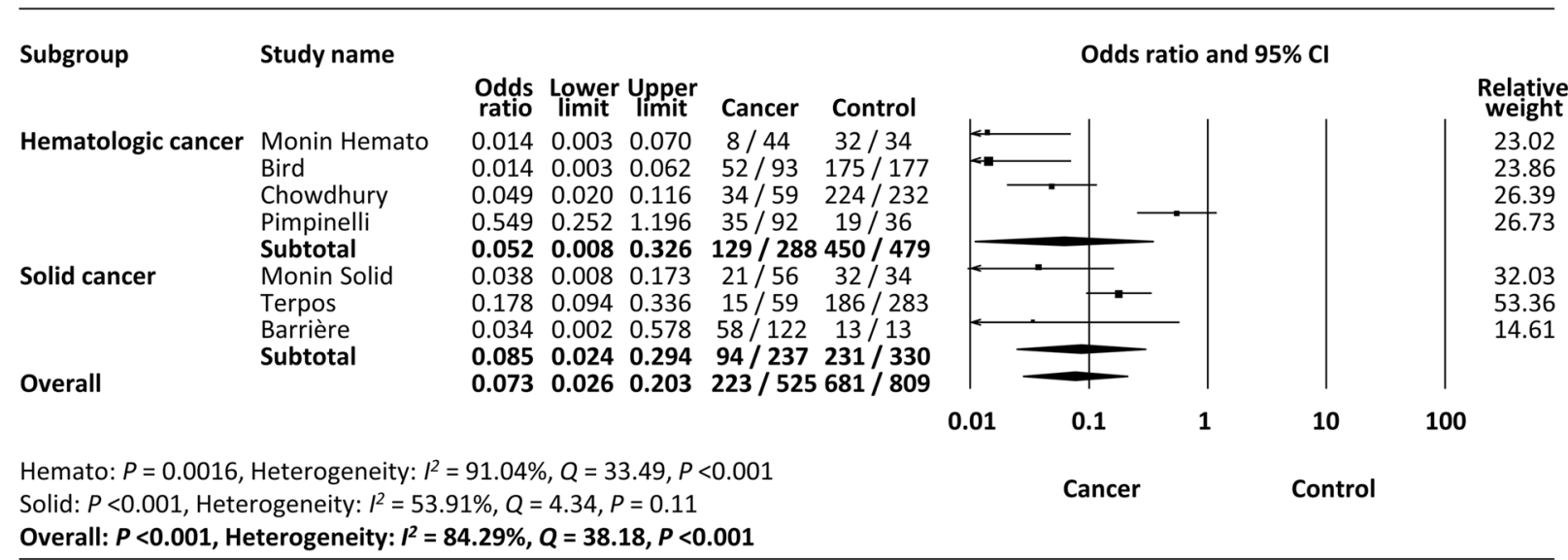

\section{B. Serologic response compared to controls after two doses of vaccine}

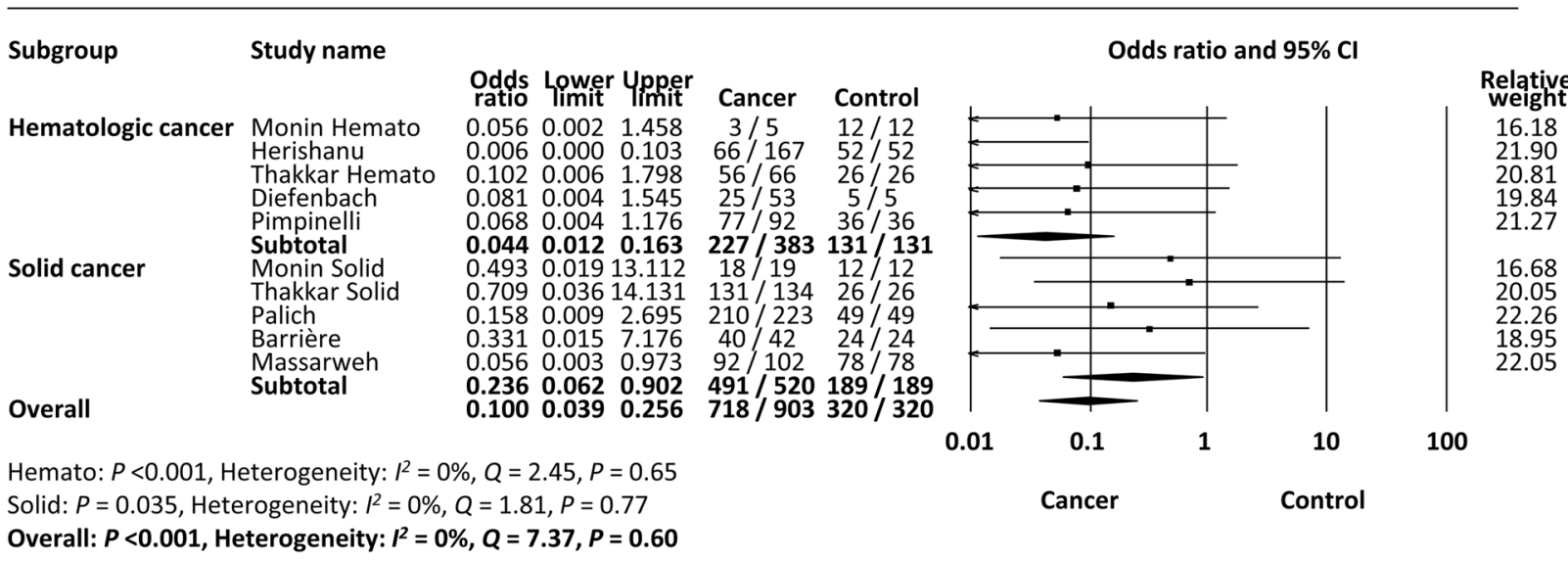

Fig. 3 A Meta-analysis of serological response compared to controls after one dose of vaccine. B Meta-analysis of serological response compared to controls after two doses of vaccine

long-term changes in immune parameters including $\mathrm{B}$ and $\mathrm{T}$ cell functions that increases the risk of various infections as well as hampers the response to vaccines [39]. They might also have a dysfunctional immune responses to infections secondary to immunotherapeutics, such as programmed cell death 1 or programmed cell death ligand 1 inhibitors, or chimeric antigen receptor (CAR)-modified T cell therapy [38]. Furthermore, patients with cancer are often older and carry comorbidities placing them at greater risk for morbidity and mortality due to COVID-19 [40].

Due to the lack of effective therapies to treat COVID19 , it is important to know the effectiveness of COVID19 vaccines in patients with cancer. Roeker et al. reported that seroconversion of anti-SARS-CoV-2 antibody after two doses of COVID-19 vaccine was 52\% in leukemia patients whereas Massarweh et al. reported a 90\% seroconversion rate in solid cancer patients [28, 30]. A majority of studies have reported an attenuated response in patients with cancer, but the seroconversion rates varied largely and most studies were of small sample sizes. Therefore, it was important to integrate findings across studies to determine the serologic response rate to COVID-19 vaccination in patients with both hematologic and solid cancers.

Shroti et al. reported that nearly all patients developed antibodies after one or two doses of BNT162b2 or ChAdOx1 nCoV-19 vaccines (96.42\% and $99.08 \%$, respectively). However, they reported that elderly people and those with comorbidities such as diabetes, cardiovascular 
disease, and cancer had lower antibody levels [41]. Our study showed that the proportion of patients achieving a serologic response after a single or two doses of COVID19 vaccine was $54 \%$ and $88 \%$, respectively, which are much lower than the rates reported by Shroti et al. Among studies that included control patients without cancer, the OR of achieving serologic response among patients with cancer was significantly lower after the first and second dose. The results of our study are consistent with recent metaanalyses reporting lower response rates in hematological cancers vs. solid cancers or controls [42, 43].

The proportion of patients on anti-cancer therapy and older age were factors associated with lower vaccine response rates after the first dose whereas diagnosis of hematologic cancer was the only factor associated with lower response after the second dose. This supports the recommendations to prioritize cancer patients for additional measures such as booster vaccinations. We also analyzed vaccine response in different types of cancer as well as in patients receiving different treatments. Patients with CLL and lymphoma, which are mainly of B-cell origin, had lower serologic response rates compared to MM, AML/CML and MPM patients and therapies such as CAR-T therapy, anti-CD20 therapy, and kinase inhibitors were associated with lower rates. In contrast, most solid cancers had serologic response rates in $80-95 \%$ range after two doses of vaccine and therapies mostly used for these conditions such as chemotherapy, immune check-point inhibitors, and hormonal therapy had higher response rates.

\section{Limitations}

Ten months have passed since the UK first approved BNT162b2, but available studies in cancer patients was still limited. There are currently 9 different vaccines on the global market, but nearly all of the included studies used only mRNA vaccines: either BNT162b2 (or mRNA1273 , and only a few studies included ChAdOx1 nCoV19 or AD26.COV2.S in a small proportion of patients. Further research is needed whether the results of our study can be generalized to other types of vaccines. We assessed humoral responses to vaccination, but the extent to which cell-mediated immunity, such as spikespecific $\mathrm{T}$ cell response, is involved remains unclear. However, recent real-world studies have shown that antibody levels are associated/predictive of infection risk and that immunosuppressed patients were at risk for breakthrough infections [44, 45]. We undertook subgroup analyses according to different diseases or therapies, but the number of studies reporting detailed data were limited. Included studies mainly used one of the three commercially available antibody tests (Roche, DiaSorin, or Abbott), which all have excellent sensitivity (98-100\%)
[46]. As a result, they correlate well with each other in terms of seroprevalence. Some early studies delayed the timing of the second dose, but all of the studies included in our meta-analysis for the assessment following two doses administered the second dose without delay. Serologic response rates were similar after two doses of BNT162b2 or mRNA-1273 vaccines, but further studies are needed to assess the difference in degree of waning antibody levels in cancer patients [47]. Furthermore, we were not able to assess the vaccine effectiveness in preventing infections or hospitalizations in cancer patients.

\section{Conclusion}

In the present comprehensive meta-analysis, we analyzed the rate of seroconversion to COVID-19 vaccines in patients with cancer. Our meta-analysis demonstrated that $54 \%$ and $88 \%$ of patients with cancer achieved a serologic response after one and two doses of COVID19 vaccine, respectively, which were statistically lower compared to controls. Cancer patients should receive the series of two dose vaccines without delay and should continue to follow safety measures including mask-wearing after vaccination. Certain conditions and therapy were associated with lower response rates, so further studies assessing optimal prophylactic strategy in patients with cancer will be warranted.

\section{Supplementary Information}

The online version contains supplementary material available at https://doi. org/10.1186/s13045-022-01233-3.

Additional file 1. Supplementary Figure 1. Funnel plot, sensitivity analyses, and subgroup analyses for serologic response after one dose of vaccine. Supplementary Figure 2. Funnel plot, sensitivity analyses, and subgroup analyses for serologic response after two doses of vaccine. Supplementary Figure 3. Funnel plots of studies included in meta-analyses of comparison of serologic response after one and two doses of vaccine compared to controls.

Acknowledgements

Not applicable.

Authors' contributions

A.S.; designed study, collected and analyzed data, drafted manuscript, full responsibility for the integrity of the work as a whole, from inception to published article, A.L.; collected and analyzed data, D.M.; data interpretation and drafted manuscript. All authors read and approved the final manuscript.

Funding

None.

Availability of data and materials Not applicable.

\section{Declarations}

Ethics approval and consent to participate Not applicable. 


\section{Consent for publication}

Not applicable.

\section{Competing interests}

The authors declare that they have no competing interests.

Received: 25 December 2021 Accepted: 27 January 2022 Published online: 05 February 2022

\section{References}

1. Chen N, Zhou M, Dong X, Qu J, Gong F, Han Y, et al. Epidemiological and clinical characteristics of 99 cases of 2019 novel coronavirus pneumonia in Wuhan, China: a descriptive study. Lancet. 2020;395(10223):507-13. https://doi.org/10.1016/S0140-6736(20)30211-7.

2. Huang C, Wang Y, Li X, Ren L, Zhao J, Hu Y, et al. Clinical features of patients infected with 2019 novel coronavirus in Wuhan, China. Lancet. 2020;395(10223):497-506. https://doi.org/10.1016/S0140-6736(20) 30183-5.

3. Wang J, Sato T, Sakuraba A. Coronavirus disease 2019 (COVID-19) meets obesity: strong association between the global overweight population and COVID-19 mortality. J Nutr. 2021;151(1):9-10. https://doi.org/10. 1093/jn/nxaa375.

4. Mehta V, Goel S, Kabarriti R, Cole D, Goldfinger M, Acuna-Villaorduna A, et al. Case fatality rate of cancer patients with COVID-19 in a New York Hospital System. Cancer Discov. 2020;10(7):935-41. https://doi.org/10 1158/2159-8290.CD-20-0516.

5. Statistics NCfH. 2019 2021.9.10. National Health Interview Survey. https:// www.cdc.gov/nchs/nhis/about_nhis.htm. 2021.9.10.

6. Siegel RL, Miller KD, Jemal A. Cancer statistics, 2019. CA Cancer J Clin. 2019;69(1):7-34. https://doi.org/10.3322/caac.21551.

7. Al-Quteimat OM, Amer AM. The impact of the COVID-19 pandemic on cancer patients. Am J Clin Oncol. 2020;43(6):452-5. https://doi.org/10. 1097/COC.0000000000000712.

8. Polack FP, Thomas SJ, Kitchin N, Absalon J, Gurtman A, Lockhart S, et al. Safety and efficacy of the BNT162b2 mRNA Covid-19 vaccine. N Engl J Med. 2020;383(27):2603-15. https://doi.org/10.1056/NEJMoa2034577.

9. Jackson LA, Anderson EJ, Rouphael NG, Roberts PC, Makhene M, Coler RN, et al. An mRNA vaccine against SARS-CoV-2-preliminary report. N Engl J Med. 2020;383(20):1920-31. https://doi.org/10.1056/NEJMoa2022 483.

10. Desai A, Gainor JF, Hegde A, Schram AM, Curigliano G, Pal S, et al. COVID19 vaccine guidance for patients with cancer participating in oncology clinical trials. Nat Rev Clin Oncol. 2021;18(5):313-9. https://doi.org/10. 1038/s41571-021-00487-z

11. Grohskopf LA, Alyanak E, Broder KR, Walter EB, Fry AM, Jernigan DB. Jernigan DB 2019 prevention and control of seasonal influenza with vaccines: recommendations of the advisory committee on immunization practices-United States, 2019-20 influenza season. MMWR Recomm Rep. 2019;68(3):1-21. https://doi.org/10.15585/mmwr.rr6803a1.

12. Keam B, Kang CK, Jun KI, Moon SM, Suh KJ, Lee D-W, et al. Immunogenicity of influenza vaccination in patients with cancer receiving immune checkpoint inhibitors. Clin Infect Dis. 2019;71(2):422-5. https://doi.org/10. 1093/cid/ciz1092.

13. Thakkar A, Gonzalez-Lugo JD, Goradia N, Gali R, Shapiro LC, Pradhan $\mathrm{K}$, et al. Seroconversion rates following COVID-19 vaccination among patients with cancer. Cancer Cell. 2021. https://doi.org/10.1016/j.ccell. 2021.06.002.

14. Addeo A, Shah PK, Bordry N, Hudson RD, Albracht B, Di Marco M, et al. Immunogenicity of SARS-CoV-2 messenger RNA vaccines in patients with cancer. Cancer Cell. 2021;39(8):1091-8.e2. https://doi.org/10.1016/j.ccell. 2021.06.009.

15. Moher D, Liberati A, Tetzlaff J, Altman DG, Group P. Preferred reporting items for systematic reviews and meta-analyses: the PRISMA statement. J Clin Epidemiol. 2009;62(10):1006-12. https://doi.org/10.1016/j.jclinepi. 2009.06.005.

16. Booth A. PROSPERO's progress and activities 2012/13. Syst Rev. 2013;2:111. https://doi.org/10.1186/2046-4053-2-111.

17. Aromataris E MZ. 2020 JBI Manual for Evidence Synthesis. JBI, 2020. https://synthesismanual.jbi.global.
18. Guyatt GH, Oxman AD, Vist G, Kunz R, Brozek J, Alonso-Coello P, et al. GRADE guidelines: 4. rating the quality of evidence-study limitations (risk of bias). J Clin Epidemiol. 2011;64(4):407-15. https://doi.org/10.1016/j.jclin epi.2010.07.017.

19. Higgins JP, Thompson SG, Deeks JJ, Altman DG. Measuring inconsistency in meta-analyses. BMJ. 2003;327(7414):557-60. https://doi.org/10.1136/ bmj.327.7414.557.

20. Higgins J, Green S. Cochrane handbook for systematic reviews of interventions, Version 5.1. 0. London: The Cochrane Collaboration 2011.

21. Begg CB, Mazumdar M. Operating characteristics of a rank correlation test for publication bias. Biometrics. 1994;50(4):1088-101.

22. Egger M, Davey Smith G, Schneider M, Minder C. Bias in meta-analysis detected by a simple, graphical test. BMJ. 1997;315(7109):629-34.

23. Clyne B, Walsh KA, O'Murchu E, Sharp MK, Comber L, Keigh KOB, et al. Using preprints in evidence synthesis: commentary on experience during the COVID-19 pandemic. J Clin Epidemiol. 2021. https://doi.org/10.1016/j. jclinepi.2021.05.010.

24. Harrington P, Doores KJ, Radia D, O'Reilly A, Lam HPJ, Seow J, et al. Single dose of BNT162b2 mRNA vaccine against severe acute respiratory syndrome coronavirus-2 (SARS-CoV-2) induces neutralising antibody and polyfunctional T-cell responses in patients with chronic myeloid leukaemia. Br J Haematol. 2021. https://doi.org/10.1111/bjh.17568.

25. Monin L, Laing AG, Muñoz-Ruiz M, McKenzie DR, del Molino del Barrio I, Alaguthurai T, et al. Safety and immunogenicity of one versus two doses of the COVID-19 vaccine BNT162b2 for patients with cancer: interim analysis of a prospective observational study. Lancet Oncol. 2021;22(6):765-78. https://doi.org/10.1016/S1470-2045(21)00213-8.

26. Herishanu Y, Avivi I, Aharon A, Shefer G, Levi S, Bronstein Y, et al. Efficacy of the BNT162b2 mRNA COVID-19 vaccine in patients with chronic lymphocytic leukemia. Blood. 2021;137(23):3165-73. https://doi.org/10.1182/ blood.2021011568.

27. Pimpinelli F, Marchesi F, Piaggio G, Giannarelli D, Papa E, Falcucci P, et al. Fifth-week immunogenicity and safety of anti-SARS-CoV-2 BNT162b2 vaccine in patients with multiple myeloma and myeloproliferative malignancies on active treatment: preliminary data from a single institution. J Hematol Oncol. 2021;14(1):81. https://doi.org/10.1186/ s13045-021-01090-6.

28. Massarweh A, Eliakim-Raz N, Stemmer A, Levy-Barda A, Yust-Katz S, Zer A, et al. Evaluation of seropositivity following BNT162b2 messenger RNA vaccination for SARS-CoV-2 in patients undergoing treatment for cancer. JAMA Oncol. 2021. https://doi.org/10.1001/jamaoncol.2021.2155.

29. Bird S, Panopoulou A, Shea RL, Tsui M, Saso R, Sud A, et al. Response to first vaccination against SARS-CoV-2 in patients with multiple myeloma. Lancet Haematol. 2021. https://doi.org/10.1016/s2352-3026(21)00110-1.

30. Roeker LE, Knorr DA, Thompson MC, Nivar M, Lebowitz S, Peters N, et al. COVID-19 vaccine efficacy in patients with chronic lymphocytic leukemia. Leukemia. 2021. https://doi.org/10.1038/s41375-021-01270-w.

31. Palich R, Veyri M, Vozy A, Marot S, Gligorov J, Benderra MA, et al. High seroconversion rate but low antibody titers after two injections of BNT162b2 (Pfizer-BioNTech) vaccine in patients treated by chemotherapy for solid cancers. Ann Oncol. 2021. https://doi.org/10.1016/j.annonc.2021. 06.018 .

32. Terpos E, Zagouri F, Liontos M, Sklirou AD, Koutsoukos K, Markellos C, et al. Low titers of SARS-CoV-2 neutralizing antibodies after first vaccination dose in cancer patients receiving checkpoint inhibitors. J Hematol Oncol. 2021;14(1):86. https://doi.org/10.1186/s13045-021-01099-x.

33. Barrière J, Chamorey E, Adjtoutah Z, Castelnau O, Mahamat A, Marco S, et al. Impaired immunogenicity of BNT162b2 anti-SARS-CoV-2 vaccine in patients treated for solid tumors. Ann Oncol. 2021;32(8):1053-5. https:// doi.org/10.1016/j.annonc.2021.04.019.

34. Chowdhury O, Bruguier H, Mallett G, Sousos N, Crozier K, Allman C, et al. Impaired antibody response to COVID-19 vaccination in patients with chronic myeloid neoplasms. Br J Haematol. 2021. https://doi.org/10.1111/ bjh.17644.

35. Agha M, Blake M, Chilleo C, Wells A, Haidar G. Suboptimal response to COVID-19 mRNA vaccines in hematologic malignancies patients. Cold Spring Harbor Laboratory; 2021.

36. Diefenbach C, Caro J, Koide A, Grossbard M, Goldberg JD, Raphael B, et al. Impaired humoral immunity to SARS-CoV-2 vaccination in non-hodgkin lymphoma and CLL patients. medRxiv. 2021. https://doi.org/10.1101/ 2021.06.02.21257804. 
37. Kuderer NM, Choueiri TK, Shah DP, Shyr Y, Rubinstein SM, Rivera DR, et al. Clinical impact of COVID-19 on patients with cancer (CCC19): a cohort study. The Lancet. 2020;395(10241):1907-18. https://doi.org/10.1016/ S0140-6736(20)31187-9.

38. Hill JA, Seo SK. How I prevent infections in patients receiving CD19targeted chimeric antigen receptor T cells for B-cell malignancies. Blood. 2020;136(8):925-35. https://doi.org/10.1182/blood.2019004000.

39. Verma R, Foster RE, Horgan K, Mounsey K, Nixon H, Smalle N, et al. Lymphocyte depletion and repopulation after chemotherapy for primary breast cancer. Breast Cancer Res. 2016;18(1):10. https://doi.org/10.1186/ s13058-015-0669-x.

40. Miyashita H, Mikami T, Chopra N, Yamada T, Chernyavsky S, Rizk D, et al. Do patients with cancer have a poorer prognosis of COVID-19? An experience in New York City. Ann Oncol. 2020;31 (8):1088-9. https://doi.org/10. 1016/j.annonc.2020.04.006.

41. Shrotri M, Fragaszy E, Geismar C, Nguyen V, Beale S, Braithwaite I, et al. Spike-antibody responses to ChAdOx1 and BNT162b2 vaccines by demographic and clinical factors (Virus Watch study). medRxiv. 2021. https:// doi.org/10.1101/2021.05.12.21257102.

42. Becerril-Gaitan A, Vaca-Cartagena BF, Ferrigno AS, Mesa-Chavez F, Barrientos-Gutiérrez T, Tagliamento M, et al. Immunogenicity and risk of Severe Acute Respiratory Syndrome Coronavirus 2 (SARS-CoV-2) infection after Coronavirus Disease 2019 (COVID-19) vaccination in patients with cancer: a systematic review and meta-analysis. Eur J Cancer. 2022;160:243-60. https://doi.org/10.1016/j.ejca.2021.10.014.

43. Gagelmann N, Passamonti F, Wolschke C, Massoud R, Niederwieser C, Adjallé R, et al. Antibody response after vaccination against SARS-CoV-2 in adults with haematological malignancies: a systematic review and meta-analysis. Haematologica. 2021. https://doi.org/10.3324/haematol. 2021.280163.

44. Khoury DS, Cromer D, Reynaldi A, Schlub TE, Wheatley AK, Juno JA, et al. Neutralizing antibody levels are highly predictive of immune protection from symptomatic SARS-CoV-2 infection. Nat Med. 2021;27(7):1205-11. https://doi.org/10.1038/s41591-021-01377-8.

45. Bergwerk M, Gonen T, Lustig Y, Amit S, Lipsitch M, Cohen C, et al. Covid-19 breakthrough infections in vaccinated health care workers. N Engl J Med. 2021;385(16):1474-84. https://doi.org/10.1056/NEJMoa2109072.

46. Di Meo A, Miller JJ, Fabros A, Brinc D, Hall V, Pinzon N, et al. Evaluation of three anti-SARS-CoV-2 serologic immunoassays for post-vaccine response. J Appl Lab Med. 2021. https://doi.org/10.1093/jalm/jfab087.

47. Shrotri M, Navaratnam AMD, Nguyen V, Byrne T, Geismar C, Fragaszy $\mathrm{E}$, et al. Spike-antibody waning after second dose of BNT162b2 or ChAdOx1. Lancet. 2021;398(10298):385-7. https://doi.org/10.1016/S01406736(21)01642-1.

\section{Publisher's Note}

Springer Nature remains neutral with regard to jurisdictional claims in published maps and institutional affiliations.

Ready to submit your research? Choose BMC and benefit from:

- fast, convenient online submission

- thorough peer review by experienced researchers in your field

- rapid publication on acceptance

- support for research data, including large and complex data types

- gold Open Access which fosters wider collaboration and increased citations

- maximum visibility for your research: over 100M website views per year

At BMC, research is always in progress.

Learn more biomedcentral.com/submissions 